\title{
Matrix product state of multi-time correlations
}

\author{
Katja Klobas $^{1}$, Matthieu Vanicat ${ }^{1}$, Juan P. Garrahan ${ }^{2,3}$ and \\ Tomaž Prosen ${ }^{1}$ \\ ${ }^{1}$ Department of Physics, Faculty of Mathematics and Physics, University of \\ Ljubljana, Ljubljana, Slovenia \\ ${ }^{2}$ School of Physics and Astronomy, University of Nottingham, Nottingham \\ NG7 2RD, United Kingdom \\ ${ }^{3}$ Centre for the Mathematics and Theoretical Physics of Quantum Non-equilibrium \\ Systems, University of Nottingham, Nottingham NG7 2RD, United Kingdom
}

\begin{abstract}
For an interacting spatio-temporal lattice system we introduce a formal way of expressing multi-time correlation functions of local observables located at the same spatial point with a time state, i.e. a statistical distribution of configurations observed along a time lattice. Such a time state is defined with respect to a particular equilibrium state that is invariant under space and time translations. The concept is developed within the Rule 54 reversible cellular automaton, for which we explicitly construct a matrix product form of the time state, with matrices that act on the 3-dimensional auxiliary space. We use the matrix-product state to express equal-space time-dependent density-density correlation function, which, for special maximum-entropy values of equilibrium parameters, agrees with the previous results. Additionally, we obtain an explicit expression for the probabilities of observing all multi-time configurations, which enables us to study distributions of times between consecutive excitations and prove the absence of decoupling of timescales in the Rule 54 model.
\end{abstract}

\section{Introduction}

When discussing locally interacting models in statistical mechanics, the central problem is the computation of time-dependent correlation functions of local observables, especially when studying non-equilibrium dynamics, transport, thermalization and alike. Multi-time correlation functions, in particular, are crucial for the understanding of complex dynamics that occurs in systems displaying metastability, such as glass formers (for reviews, see e.g. [1-3|). However, obtaining an exact form or at least asymptotic behaviour of such correlation functions is usually out of the scope of the current analytical methods, even for the simplest interacting systems. Sometimes even partial knowledge of multi-time correlation functions of local observables located at the same point can provide useful insights. For example, when studying the transport of conserved quantities, knowing the multi-time equal-space density-density correlation function provides the scaling behaviour of the central (heat) peak within the hydrodynamic description [4]. On other hand, multi-time equal-space correlation functions can be 


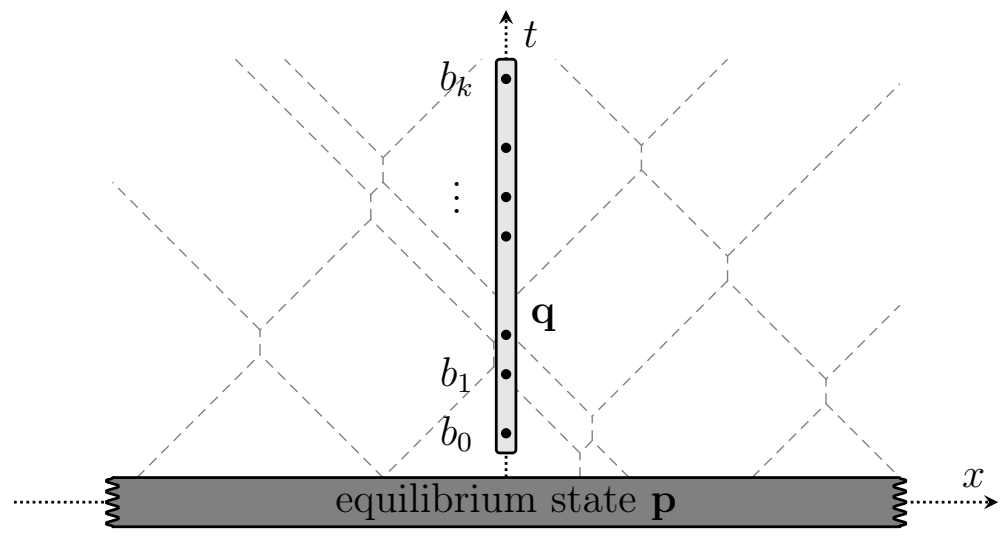

Figure 1. Schematic picture of the setup. We are interested in probability distribution $\mathbf{q}$ of the configurations $\left(b_{0}, b_{1}, \cdots, b_{k}\right)$ at the center of the chain, $x=0$, and different times $t$, while the system is in the equilibrium state p. Intuitively, we can understand this as keeping track of the particles that pass through the origin. Since they are interacting with each other, this is generally not a trivial task and the computational complexity of $\mathbf{q}$ might still be growing exponentially with time.

viewed as expectation values of a specific class of observables that are nonlocal in time, but local in space.

Specifically, we will be interested in the multi-point correlation functions of singlesite (ultralocal) observables $a_{j}$ located at the same spatial point $x=0$ but at different times $t_{j}$

$$
C_{a_{1}, a_{2}, \ldots, a_{k}}\left(t_{1}, t_{2}, \ldots, t_{k}\right)=\left\langle a_{1}\left(0, t_{1}\right) a_{2}\left(0, t_{2}\right) \cdots a_{k}\left(0, t_{k}\right)\right\rangle_{\mathbf{p}},
$$

where $\mathbf{p}$ denotes an equilibrium state (which is, by definition, time-translation invariant). The computational complexity of the time evolution of local observables in interacting systems generically grows exponentially even within classical mechanics 1 but since all of them are positioned at the same point, one expects that a lot of information contained in the time propagated observables $a_{j}(x, t)$ is not necessary for knowing the expectation value (1). Therefore we propose that, at least in certain cases, the computational complexity could be reduced by introducing a time state $\mathbf{q}(\mathbf{p})$, i.e. the probability distribution of observing configurations in time $\left(b_{0}, b_{1}, b_{2}, \ldots, b_{k}\right)$ if the system is in the equilibrium state $\mathbf{p}$, as is schematically shown in Figure 1. Here $b_{j}$ label a complete set of ultralocal observables.

In the paper we show that it is possible to construct an explicit nontrivial matrixproduct representation of such states in the case of Rule 54 reversible cellular automaton (RCA54), introduced in [5]. RCA54 is a classical deterministic model in discrete spacetime of particles that move with constant velocities and interact pairwise by shifting for one site when scattering. The model is also closely related to the automaton ERCA 250R, as introduced by Takesue [6], and to the Floquet version of Fredrickson-Andersen model [7 9]. The simplicity of the model allows for a lot of exact results, such as the $\ddagger$ By computational complexity we refer to the cost of computing the propagated observable exactly, not approximating it (numerically). 
non-equilibrium steady state of the system coupled to stochastic reservoirs [10,11, as well the computation of the most relevant (long lived) decay modes [12], and exact treatment of large deviation statistics [13|. An explicit matrix-product representation of the complete time evolution of local observables was found in [14], and was afterwards generalized to the time evolution of local density matrices in the quantum version of the model 15.

The paper is structured as follows; in section 2 we define the model and introduce a two-parameter family of equilibrium states. They are expressed in terms of a matrix-product ansatz (MPA), with matrices that obey the cubic cancellation relation introduced in 12 . We express asymptotic probabilities of observing finite subconfigurations in the limit of large system sizes and show that the probabilities of observing solitons are statistically independent. Section 3 contains the main result of the paper, namely the explicit construction of the time state. Due to the statistical independence of solitons, the construction reduces to the problem of keeping track of all the quasi-particles that pass through the site 0 . This is achieved by introducing matrices that act on a 3-dimensional auxiliary space and expressing the time state in an MPA form. In sections 4 and 5 we briefly discuss examples of correlation functions and expectation values that can be exactly expressed using the MPA form of the time state. Finally, in section 6 we finish with closing remarks.

\section{Rule 54 reversible cellular automaton}

\subsection{Definition of the dynamics}

The model is a deterministic cellular automaton defined on a chain of even length $2 n$, with every site being either occupied or empty. The configuration of the system at time $t$ is given by the string of bits $\left(s_{1}^{t}, s_{2}^{t}, \ldots, s_{2 n}^{t}\right)$, where $s_{j}^{t}=0$ if the site $j$ is empty at time $t$ and $s_{j}^{t}=1$ otherwise. The time evolution consists of two time steps, in the first one only the values on even sites are changed, while in the second one the odd sites are updated,

$$
s_{x}^{t+1}=\left\{\begin{array}{lll}
\chi\left(s_{x-1}^{t}, s_{x}^{t}, s_{x+1}^{t}\right) ; & x+t \equiv 0 & (\bmod 2), \\
s_{x}^{t} ; & x+t \equiv 1 & (\bmod 2),
\end{array}\right.
$$

where we assume periodic boundaries, $s_{2 n+k}^{t} \equiv s_{k}^{t}$. The local update map $\chi$ is given by the Rule 54 (RCA54) of Bobenko et. al., as introduced in [5],

$$
\chi\left(s_{1}, s_{2}, s_{3}\right)=\left(s_{1}+s_{2}+s_{3}+s_{1} s_{3}\right) \quad(\bmod 2) .
$$

It is convenient to imagine the chain to have a zig-zag shape, as schematically shown in Figure 2. Then, the local update rule can be expressed graphically by representing occupied sites by black and empty sites by white squares,
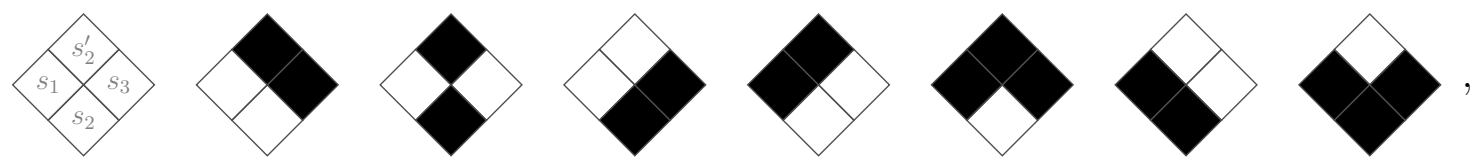


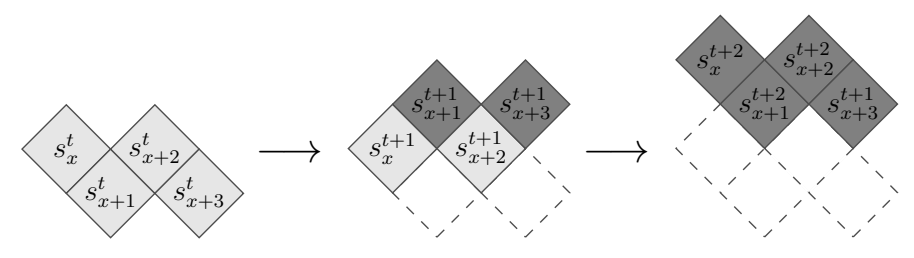

Figure 2. Schematic representation of the lattice geometry and time evolution. Only half of the sites are being updated at the same time (2), therefore it is convenient to imagine the chain to have a zig-zag shape. At each time step, the sites positioned at the bottom (e. g. $s_{x+1}^{t}$ and $s_{x+3}^{t}$ on the left most picture) are propagated, while the top sites $\left(s_{x}^{t}\right.$ and $\left.s_{x+2}^{t}\right)$ do not change. The new value depends on the values of the three consecutive sites as described in (2) and (4).

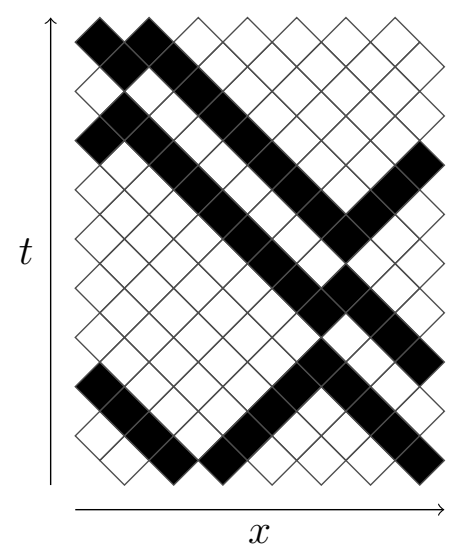

Figure 3. An example of the time evolution. We start with a configuration of 14 sites and evolve it according to the rules (4) with periodic boundary conditions. The black sites behave as particles that move with velocity 1 either to the left or to the right. The particles interact pairwise, by annihilating and then reappearing in the next time step (described by the 3rd, 6th and 8th diagram in (4)). This induces a delay for one site with respect to the original trajectories of the particles.

where the bottom three squares represent a configuration of three consecutive sites at time $t,\left(s_{1}, s_{2}, s_{3}\right)$, while the top square is the updated site, $s_{2}^{\prime}=\chi\left(s_{1}, s_{2}, s_{3}\right)$. An example of the time evolution induced by these rules is shown in Figure 3 . The graphical representation provides an intuitive interpretation of the dynamics; occupied sites behave as particles that move with velocity \pm 1 while undisturbed. When two oppositely moving particles meet, they interact by being delayed for one site (or one time step) with respect to their initial trajectories.

\subsection{Time evolution of probability distributions}

The states $\mathbf{p}$ are probability distributions over configurations and can be expressed as vectors in $\mathbb{R}^{2^{2 n}}$, with nonnegative components and the appropriate normalization,

$$
\mathbf{p}=\left(p_{0}, p_{1}, \ldots, p_{2^{2 n}-1}\right), \quad p_{s} \geq 0, \quad \sum_{s=0}^{2^{2 n}-1} p_{s}=1,
$$


where $p_{s}$ is the probability of observing the configuration $\left(s_{1}, s_{2}, \ldots, s_{2 n}\right)$, given by the binary representation of $s: s=\sum_{j=1}^{2 n} 2^{2 n-, j} s_{j}$. The local update rule is given in terms of the three-site permutation matrix $P$ with the elements $P_{\left(s_{1} s_{2} s_{3}\right),\left(b_{1} b_{2} b_{3}\right)}=$ $\delta_{s_{1}, b_{1}} \delta_{s_{2}, \chi\left(b_{1}, b_{2}, b_{3}\right)} \delta_{s_{3}, b_{3}}$. Explicitly,

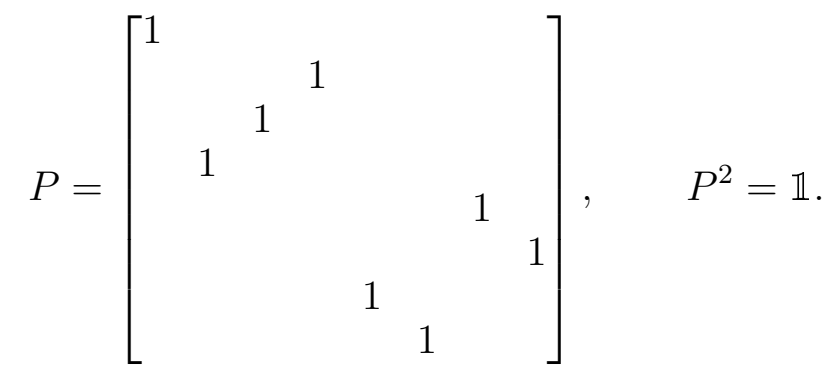

Introducing the local propagators of the triplets of neighbouring sites as $P_{x-1, x, x+1}=$ $\mathbb{1}_{2^{x-2}} \otimes P \otimes \mathbb{1}_{2^{2 n-x-1}}$, the time evolution of the state $\mathbf{p}^{t}$ can be expressed in the following way,

$$
\begin{aligned}
& \mathbf{p}^{t+1}=\left\{\begin{array}{lll}
U_{\mathrm{e}} \mathbf{p}^{t} ; & t \equiv 0 & (\bmod 2), \\
U_{\mathrm{o}} \mathbf{p}^{t} ; & t \equiv 1 & (\bmod 2),
\end{array}\right. \\
& U_{\mathrm{e}}=\prod_{x=1}^{n} P_{2 x-1,2 x, 2 x+1}, \\
& U_{\mathrm{o}}=\prod_{x=1}^{n} P_{2 x-2,2 x-1,2 x} .
\end{aligned}
$$

\subsection{Equilibrium states}

The equilibrium states should be invariant under the time evolution for whole periods of time, i.e. for even $t$. Since the time propagation is staggered, we can introduce two versions of the equilibrium state, $\mathbf{p}$ and $\mathbf{p}^{\prime}$, corresponding to the even and the odd time steps. In this case the time-invariance condition takes the following form,

$$
\mathbf{p}^{\prime}=U_{\mathrm{e}} \mathbf{p}, \quad \mathbf{p}=U_{\mathrm{o}} \mathbf{p}^{\prime}
$$

In the paper we consider the states that can be expressed in a matrix-product form similar to the stationary states introduced in 12,13 . Let $\mathbf{W}(\xi, \omega)$ and $\mathbf{W}^{\prime}(\xi, \omega)$ be vectors in the physical space,

$$
\mathbf{W}(\xi, \omega)=\left[\begin{array}{l}
W_{0}(\xi, \omega) \\
W_{1}(\xi, \omega)
\end{array}\right], \quad \mathbf{W}^{\prime}(\xi, \omega)=\left[\begin{array}{l}
W_{0}^{\prime}(\xi, \omega) \\
W_{1}^{\prime}(\xi, \omega)
\end{array}\right] .
$$

Their components are $3 \times 3$ matrices that depend on two spectral parameters $\xi$ and $\omega$; $\xi, \omega>0$,

$W_{0}(\xi, \omega)=\left[\begin{array}{lll}1 & 0 & 0 \\ \xi & 0 & 0 \\ 1 & 0 & 0\end{array}\right]=W_{0}^{\prime}(\omega, \xi), \quad W_{1}(\xi, \omega)=\left[\begin{array}{lll}0 & \xi & 0 \\ 0 & 0 & 1 \\ 0 & 0 & \omega\end{array}\right]=W_{1}^{\prime}(\omega, \xi)$.

The two sets of matrices $\mathbf{W}^{\prime}(\xi, \omega), \mathbf{W}^{\prime}(\xi, \omega)$ are mapped to each other under the exchange of the (spectral) parameters $\xi \leftrightarrow \omega$. In what follows, the explicit dependence 
on $\xi$ and $\omega$ will sometimes be suppressed to simplify the notation. The matrices obey a cubic algebraic relation

$$
P_{123} \mathbf{W}_{1} \mathbf{W}_{2}^{\prime} \mathbf{W}_{3} S=\mathbf{W}_{1} S \mathbf{W}_{2} \mathbf{W}_{3}^{\prime},
$$

where $S$ is a $3 \times 3$ matrix acting in the auxiliary space:

$$
S=\left[\begin{array}{lll}
1 & & \\
& & 1 \\
& 1 &
\end{array}\right], \quad S^{2}=\mathbb{1} .
$$

The relation, spelled out in physical components, explicitly states $W_{s_{1}} W_{\chi\left(s_{1}, s_{2}, s_{3}\right)}^{\prime} W_{s_{3}} S=$ $W_{s_{1}} S W_{s_{2}} W_{s_{3}}^{\prime}$, for any combination of $s_{1}, s_{2}, s_{3} \in\{0,1\}$. Due to the mapping between $\mathbf{W}$ and $\mathbf{W}^{\prime}$, the following dual relation also holds,

$$
P_{123} \mathbf{W}_{1}^{\prime} \mathbf{W}_{2} \mathbf{W}_{3}^{\prime} S=\mathbf{W}_{1}^{\prime} S \mathbf{W}_{2}^{\prime} \mathbf{W}_{3} .
$$

The equilibrium states can then be expressed as

$$
\mathbf{p}^{(2 n)}=\frac{1}{Z} \operatorname{tr}\left(\mathbf{W}_{1} \mathbf{W}_{2}^{\prime} \cdots \mathbf{W}_{2 n}^{\prime}\right), \quad \mathbf{p}^{\prime(2 n)}=\frac{1}{Z} \operatorname{tr}\left(\mathbf{W}_{1}^{\prime} \mathbf{W}_{2} \cdots \mathbf{W}_{2 n}\right),
$$

where $Z$ is fixed by the normalization. Using the relations $(11)$ and $(13)$, together with properties $S^{2}=\mathbb{1}$ and $P^{2}=\mathbb{1}$, it is straightforward to see that the states $\mathbf{p}^{(2 n)}$ and $\mathbf{p}^{\prime(2 n)}$ are mapped to each other under the time propagation,

$$
\mathbf{p}^{(2 n)}=U_{\mathrm{o}} \mathbf{p}^{\prime(2 n)}, \quad \mathbf{p}^{\prime(2 n)}=U_{\mathrm{e}} \mathbf{p}^{(2 n)},
$$

which implies their stationarity. Note that the exchange of the parameters $\xi \leftrightarrow \omega$ shifts the state for one time step. Furthermore, the exchange of parameters also corresponds to the shift for one lattice site. Explicitly,

$$
\eta\left(\mathbf{p}^{(2 n)}\right)=\mathbf{p}^{\prime(2 n)}, \quad \eta\left(\mathbf{p}^{\prime(2 n)}\right)=\mathbf{p}^{(2 n)},
$$

where $\eta$ is the one-lattice-site shift operator, given in terms of a $2^{2 n} \times 2^{2 n}$ matrix $\eta_{\left(s_{1} s_{2} \ldots s_{2 n}\right),\left(b_{1} b_{2} \ldots b_{2 n}\right)}=\delta_{s_{2}, b_{1}} \delta_{s_{3}, b_{2}} \delta_{s_{4}, b_{3}} \cdots \delta_{s_{2 n}, b_{2 n-1}} \delta_{s_{1}, b_{2 n}}$. It can be argued that 14 provides a two-parameter $\left(\xi, \omega \in \mathbb{R}_{\geq 0}\right)$ family of finitely correlated equilibrium states of the model, a kind of a caricature of generalized Gibbs states.

\subsection{Asymptotic equilibrium distributions of shorter configurations}

The equilibrium distribution on a smaller subchain of length $2 m, m \leq n$, can be expressed by summing over all the other sites

$$
\mathbf{p}_{[1,2 m]}^{(2 n)}=\frac{\operatorname{tr}\left(\mathbf{W}_{1} \mathbf{W}_{2}^{\prime} \mathbf{W}_{3} \cdots \mathbf{W}_{2 m}^{\prime} T^{n-m}\right)}{\operatorname{tr} T^{n}},
$$

with the transfer matrix $T=\left(W_{0}+W_{1}\right)\left(W_{0}^{\prime}+W_{1}^{\prime}\right)$ taking the form

$$
T=\left[\begin{array}{ccc}
1+\xi \omega & \omega & \xi \\
1+\xi & \xi \omega & \xi \\
1+\omega & \omega & \xi \omega
\end{array}\right]
$$


Fixing the subchain length $m$ while taking the thermodynamic limit $n \rightarrow \infty$ this reduces to

$$
\mathbf{p}_{[1,2 m]}=\lim _{n \rightarrow \infty} \mathbf{p}_{[1,2 m]}^{(2 n)}=\lambda^{-m} \frac{\left\langle l\left|\mathbf{W}_{1} \mathbf{W}_{2}^{\prime} \mathbf{W}_{3} \cdots \mathbf{W}_{2 m}^{\prime}\right| r\right\rangle}{\langle l \mid r\rangle},
$$

where $\lambda$ is the leading eigenvalue of $T$,

$$
\begin{aligned}
& \lambda(\xi, \omega)=\frac{1}{3}\left(1+3 \xi \omega+\left(\frac{\Delta}{2}\right)^{1 / 3}+(1+3 \xi)(1+3 \omega)\left(\frac{2}{\Delta}\right)^{1 / 3}\right) \\
& \Delta=2+9(\xi+\omega)+27 \xi \omega(2+\xi+\omega)+\sqrt{27(\xi-\omega)^{2}(9 \xi \omega(3 \xi \omega-2)-4(\xi+\omega)-1)}
\end{aligned}
$$

and $\langle l|| r$,$\rangle are the corresponding left and right (unnormalized) eigenvectors,$

$$
|l\rangle=\left[\begin{array}{c}
(\lambda-\xi \omega)^{2}-\xi \omega \\
\omega(\lambda-\xi \omega+\xi) \\
\xi(\lambda-\xi \omega+\omega)
\end{array}\right], \quad|r\rangle=\left[\begin{array}{c}
\omega(\lambda-\xi \omega+\xi) \\
(\lambda-\xi \omega)^{2}-\lambda-\xi \\
\omega(\lambda-\xi \omega+\omega)
\end{array}\right] .
$$

Note that the leading eigenvalue $\lambda(\xi, \omega)$ is the largest solution of the cubic equation

$$
\lambda^{3}-\lambda^{2}(1+3 \xi \omega)-\lambda(\xi+\omega+\xi \omega(1-3 \xi \omega))-\xi \omega(1-\xi \omega)^{2}=0 .
$$

It is larger than 1 and appears as a single (isolated) real root for all non-negative values of the parameters $\xi, \omega$.

The expression (19) holds for all finite subsections of the chain that start at odd sites at even times or even sites at odd times, i.e. the components of $\mathbf{p}$ are probabilities of observing configurations $\left(s_{x}^{t}, s_{x+1}^{t}, \ldots, s_{x+2 m-1}^{t}\right)$ if $x+t \equiv 1(\bmod 2)$ holds. $\S$ In other case, we have to exchange the role of the parameters $\xi$ and $\omega$. This can be summarized as

$$
\begin{aligned}
& p\left(s_{s_{2}} \cdots \lambda_{s_{2 m}}\right):=p_{s_{1} s_{2} \ldots s_{2 m}}:=\frac{\lambda^{-m}}{\langle l \mid r\rangle}\left\langle l\left|W_{s_{1}} W_{s_{2}}^{\prime} \cdots W_{s_{2 m}}^{\prime}\right| r\right\rangle, \\
& p\left(l_{s_{2 m}}^{\prime}\right):=p_{s_{1} s_{2} \ldots s_{2 m}}^{\prime}:=\frac{\lambda^{-m}}{\left\langle l^{\prime} \mid r^{\prime}\right\rangle}\left\langle l^{\prime}\left|W_{s_{1}}^{\prime} W_{s_{2}} \cdots W_{s_{2 m}}\right| r^{\prime}\right\rangle,
\end{aligned}
$$

where the new (primed) left/right vectors are obtained from the old (unprimed) ones by exchanging $\xi \leftrightarrow \omega:\left\langle l^{\prime}(\xi, \omega)\right|=\langle l(\omega, \xi)|$ and $\left|r^{\prime}(\xi, \omega)\right\rangle=|r(\omega, \xi)\rangle$.

The asymptotic equilibrium distribution on $2 m$ sites (19) can be alternatively understood as a steady-state solution to a specific boundary driven setup, where the bulk dynamics is given by the deterministic update rules (4), while the boundary sites change stochastically (i.e. the situation studied in $10-13 \mid$ ). In Appendix A we provide the details about the equivalent stochastic-boundary setup.

\subsection{Statistical independence of solitons}

The stationary (equilibrium) distributions $\mathbf{p}(\xi, \omega)$, Eq. (14), are characterized by statistical independence of solitons, i.e. the probability of encountering a soliton is

$\S$ Since $\mathbf{p}_{[1,2 m]}$ is well defined for any $m$, the subscript will be omitted and the exact length specified when ambiguous. In what follows, $\mathbf{p}$ will always refer to the asymptotic equilibrium distribution. 
the same everywhere, independently of the positions of other solitons. This is a consequence of the fact that the conditional probability of observing $\left(s_{2 k-1}, s_{2 k}\right)$, given the previous configuration $\left(s_{1}, s_{2}, \ldots, s_{2 k-2}\right)$, depends only on the value of the last four bits, $\left(s_{2 k-3}, s_{2 k-2}, s_{2 k-1} s_{2 k}\right)$. Explicitly,

$\frac{p_{s_{1} s_{2} \ldots s_{2 k-1} s_{2 k}}}{p_{s_{1} s_{2} \ldots s_{2 k-2}}}=\frac{p_{s_{2 k-3} s_{2 k-2} s_{2 k-1} s_{2 k}}}{p_{s_{2 k-3} s_{2 k-2}}}, \quad \frac{p_{s_{1} s_{2} \ldots s_{2 k-1} s_{2 k}}^{\prime}}{p_{s_{1} s_{2} \ldots s_{2 k-2}}^{\prime}}=\frac{p_{s_{2 k-3} s_{2 k-2} s_{2 k-1} s_{2 k}}^{\prime}}{p_{s_{2 k-3} s_{2 k-2}}^{\prime}}$.

A similar relation holds for the conditional probability of finding $\left(s_{1}, s_{2}\right)$ on the first two sites given that the sites from 3 to $2 k$ are in the configuration $\left(s_{3}, s_{4}, \ldots s_{2 k}\right)$;

$$
\frac{p_{s_{1} s_{2} \ldots s_{2 k}}}{p_{s_{3} s_{4} \ldots s_{2 k}}}=\frac{p_{s_{1} s_{2} s_{3} s_{4}}}{p_{s_{3} s_{4}}}, \quad \frac{p_{s_{1} s_{2} \ldots s_{2 k}}^{\prime}}{p_{s_{3} s_{4} \ldots s_{2 k}}^{\prime}}=\frac{p_{s_{1} s_{2} s_{3} s_{4}}^{\prime}}{p_{s_{3} s_{4}}^{\prime}} .
$$

A proof of these relations simply follows from the sparse structure of the matrices $W_{s}$, $W_{s}^{\prime}$, as is shown in Appendix B. Physically, this means that due to the pure contact (ultra local) interactions between particles the equilibrium state can be interpreted as an ideal gas of solitons.

Taking into account (24) and (25) we can now introduce probabilities of observing the left/right movers, $\left(p_{l}, p_{r}\right)$. The first parameter, $p_{l}$, is the conditional probability of observing a left mover, if we know that the neighbouring left ray does not contain a left moving soliton. This can be easily expressed in terms of equilibrium probabilities as

$$
p_{l}=\frac{p(\diamond)+p(\diamond)}{p(\diamond)}=\frac{p(\diamond)+p(\diamond)}{p(\diamond)}=\frac{\xi(\lambda+\omega(1-\xi))}{\lambda(1+\xi)+\xi(1-\xi \omega)} .
$$

Similarly, $p_{r}$ is the conditional probability of observing a right moving soliton, provided that the neighbouring right ray does not contain a right mover,

$$
p_{r}=\frac{p(\diamond)+p(\diamond)}{p(\diamond)}=\frac{p(\diamond)+p(\diamond)}{p(\diamond)}=\frac{\omega(\lambda+\xi(1-\omega))}{\lambda(1+\omega)+\omega(1-\xi \omega)} .
$$

Note that the shift for one time step or one lattice site maps $p_{r}$ to $p_{l}$ and vice versa; $p_{l} \stackrel{\xi \leftrightarrow \omega}{\longleftrightarrow} p_{r}$.

\section{Probability distributions of configurations in time}

We now proceed to the main result of the paper, namely the construction of the matrix product representation of the time state $\mathbf{q}$. The components of the probability vector $q_{b_{0} b_{1} \ldots b_{m-1}}$ are the probabilities of encountering multi-time configurations encoded by bit strings $\left(b_{0}, b_{1}, \ldots b_{m-1}\right) \in\{0,1\}^{m}$ in the middle vertical saw, while the horizontal chain configurations (say along the bottom saw) are distributed according to the equilibrium state $\mathbf{p}$ (schematically depicted in Fig. 1). Explicitly,

$$
q_{b_{0} b_{1} \ldots b_{m-1}}=\sum_{\underline{s} \equiv\left(s_{-m}, s_{-m+1}, \ldots, s_{m-1}\right)} p_{\underline{s}} \delta_{b_{0}, s_{0}^{0}} \delta_{b_{1}, s_{1}^{1}} \delta_{b_{2}, s_{0}^{2}} \delta_{b_{3}, s_{1}^{3}} \cdots \delta_{b_{m-1}, s_{\bmod (m-1,2)}^{m-1}},
$$

where $s_{k}^{t}$ is the value at site $k$ of the configuration $\underline{s}=\left(s_{-m}, s_{-m+1}, \ldots s_{m-1}\right)$ propagated for $t$ time steps, starting from the initial data $s_{k}^{0}=s_{k}$. Due to the staggering, we should 
consider the site 0 at even time steps and the site 1 at odd time steps. The last site, whether 0 or 1 , therefore depends on the parity of $m$. I.

The definition of the time-state components (28) does not explicitly rely on the particularities of the model and can be directly applied to any system, for which the time-evolved configurations $\underline{s}^{t}$ are in one-to-one correspondence with the initial configurations $\underline{s}^{0}$, i.e. any deterministic classical system with a finite configuration space. Moreover, the time-state can formally be defined for any underlying statistical state by replacing the probabilities $p_{\underline{s}}$ accordingly. However, as we show below, for our specific choice of asymptotic stationary probability distribution (23) the time-state greatly simplifies, which is not true in general.

Since the positions of the solitons in the equilibrium state $\mathbf{p}$ are statistically independent, the probability of a left (right) mover reaching the central site at time $t$ does not depend on the previously observed solitons, as long as there was no left (right) mover in the previous time step $t-1$. Therefore, the conditional probability of observing a vertical configuration $\left(b_{0}, b_{1}, b_{2}, \ldots b_{k-1}, b_{k}\right)$ given the previous configuration $\left(b_{0}, b_{1}, b_{2}, \ldots b_{k-1}\right)$, depends only on the last 4 bits. This implies a relation similar to (24) and (25),

$\frac{q_{b_{0} b_{1} \ldots b_{2 k-1}}}{q_{b_{0} b_{1} \ldots b_{2 k-2}}}=f\left(b_{2 k-4}, b_{2 k-3}, b_{2 k-2}, b_{2 k-1}\right), \quad \frac{q_{b_{0} b_{1} \ldots b_{2 k}}}{q_{b_{0} b_{1} \ldots b_{2 k-1}}}=f^{\prime}\left(b_{2 k-3}, b_{2 k-2}, b_{2 k-1}, b_{2 k}\right)$,

where we introduced two yet unknown functions of the last four bits, $f, f^{\prime}: \mathbb{Z}_{2} \times \mathbb{Z}_{2} \times$ $\mathbb{Z}_{2} \times \mathbb{Z}_{2} \rightarrow \mathbb{R}_{\geq 0}$, that have to be mapped into each other under the exchange of the spectral parameters $\xi$ and $\omega$ (or equivalently, $p_{r}$ and $p_{l}$ ),

$$
f\left(b_{1}, b_{2}, b_{3}, b_{4}\right) \stackrel{p_{l} \leftrightarrow p_{r}}{\longleftrightarrow} f^{\prime}\left(b_{1}, b_{2}, b_{3}, b_{4}\right) .
$$

To determine the functions $f, f^{\prime}$, one should first classify all the vertical configurations of 3 sites and their transitions into 4-site configurations. They can be divided into 3 different types:

- A configuration might be inaccessible, i.e. the configuration is inconsistent with the time evolution rules (4). The probability of obtaining such configurations is 0 . Such are the following configurations:

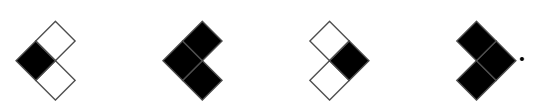

- A configuration is allowed and the local update rules deterministically determine the next bit, i.e. the conditional probability $q_{b_{k-3} b_{k-2} b_{k-1} b_{k}} / q_{b_{k-3} b_{k-2} b_{k-1}}$ of observing $b_{k}$ is either 0 or 1 . The following 3 configurations are of this type:

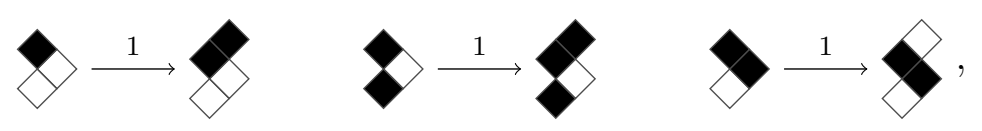

II Note also that the labels of the sites now go from $-m$ to $m-1$ and no longer from 1 to $2 m$. The convention is such, that the sites with the even labels are getting propagated in the first half step and the sites with the odd labels in the second one, irrespective of the parity of $m$. 
and analogously for the other parity of $k$ (i.e. considering the flipped configurations),

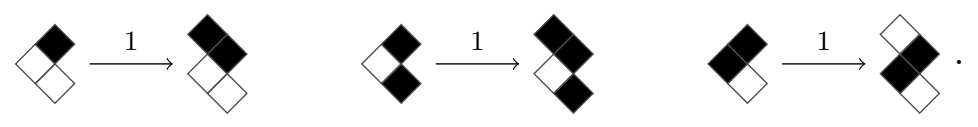

Complementary diagrams with negated $b_{k}$ are associated with conditional probability 0 .

- A configuration is allowed and both the configurations $\left(b_{k-3} b_{k-2} b_{k-1} 0\right)$ and $\left(b_{k-3} b_{k-2} b_{k-1} 1\right)$ are also allowed. These are the following:
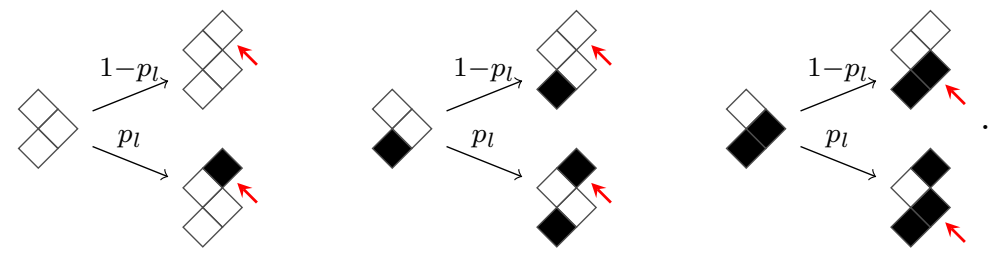

The conditional probabilities $q_{b_{k-3} b_{k-2} b_{k-1} b_{k}} / q_{b_{k-3} b_{k-2} b_{k-1}}$ correspond to the probability of a left mover (not)reaching the vertical saw (corresponding to the top/bottom option respectively) at the position indicated by the red arrows. Since the solitons in the equilibrium state are independent, the probability $p_{l}$ is given by (26). For the flipped configurations, everything works similarly, only $p_{l}$ has to be replaced with $p_{r}$ from (27),
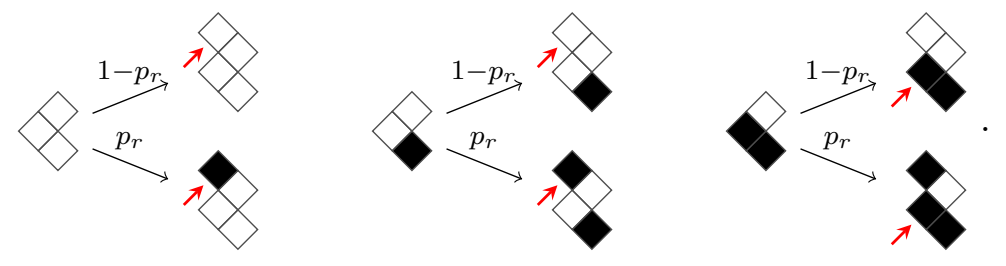

Extracting the precise values of $f\left(b_{1}, b_{2}, b_{3}, b_{4}\right)$ and $f^{\prime}\left(b_{1}, b_{2}, b_{3}, b_{4}\right)$ from the diagrams 31,35 , the probability of a vertical configuration can be explicitly expressed as

$q_{b_{0} b_{1} \ldots b_{k}}=q_{b_{0} b_{1} b_{2}} f\left(b_{0}, b_{1}, b_{2}, b_{3}\right) f^{\prime}\left(b_{1}, b_{2}, b_{3}, b_{4}\right) \cdots f^{(\prime)}\left(b_{k-1}, b_{k-2}, b_{k-1}, b_{k}\right)$,

where the last value is $f\left(b_{k-1}, b_{k-2}, b_{k-1}, b_{k}\right)$, if $k$ is odd, and $f^{\prime}\left(b_{k-1}, b_{k-2}, b_{k-1}, b_{k}\right)$ otherwise. The probabilities of configurations of length 3 can be determined by requiring $\sum_{b_{0}, b_{1}} q_{b_{0} b_{1} b_{2} b_{3} b_{4}}=q_{b_{2} b_{3} b_{4}}$.

The probability vector (36) can be efficiently encoded within an MPA by introducing a 3-dimensional auxiliary space, with basis vectors $\mid 0), \mid 1), \mid 2$ ) keeping the relevant information about the last three bits. The pairs of matrices $A_{s}\left(p_{r}, p_{l}\right), A_{s}^{\prime}\left(p_{r}, p_{l}\right)$ and left/right boundary vectors $\left(L\left(p_{r}, p_{l}\right)|| R,\left(p_{r}, p_{l}\right)\right)$ are constructed, so that the probability vector $\mathbf{q}$ is expressed as

$$
\mathbf{q}=\left(L\left|\mathbf{A}_{0} \mathbf{A}_{1}^{\prime} \mathbf{A}_{2} \cdots \mathbf{A}_{k}^{(\prime)}\right| R\right)
$$

The last matrix is either $\mathbf{A}$ or $\mathbf{A}^{\prime}$, depending on the parity of the last index $k$. The 
matrices $A_{s}\left(p_{r}, p_{l}\right)$ contain the relevant transition rates $f, g$,

$$
A_{0}=\left[\begin{array}{ccc}
1-p_{r} & 0 & 0 \\
0 & 0 & 0 \\
1 & 0 & 0
\end{array}\right], \quad A_{1}=\left[\begin{array}{ccc}
0 & p_{r} & 0 \\
0 & 0 & 1 \\
0 & 0 & 0
\end{array}\right]
$$

and the other pair of matrices is obtained by replacing $p_{r}$ with $p_{l}$,

$$
\mathbf{A}^{\prime}\left(p_{r}, p_{l}\right)=\mathbf{A}\left(p_{l}, p_{r}\right) .
$$

The left and right vectors $(L|| R$,$) are the left and the right eigenvector of the$ matrix $\mathcal{T}=\left(A_{0}+A_{1}\right)\left(A_{0}^{\prime}+A_{1}^{\prime}\right)$ that correspond to the leading eigenvalue (which is 1), namely

$$
\left(L\left|=\frac{1}{1+p_{r}+p_{l}}\left[\begin{array}{lll}
1 & p_{l} & p_{r}
\end{array}\right], \quad\right| R\right)=\left[\begin{array}{l}
1 \\
1 \\
1
\end{array}\right] .
$$

Additionally, this choice of $\mid R$ ) works for both parities of $k$, since $\mid R$ ) is an eigenvector corresponding to the eigenvalue 1 of both $A_{0}+A_{1}$ and $A_{0}^{\prime}+A_{1}^{\prime}$. The normalization of the boundary vectors is determined by the normalization condition of the probability vector $\mathbf{q}$,

$$
\sum_{b_{0}, b_{1}, b_{2}, \ldots, b_{k}} q_{b_{0} b_{1} b_{2} \ldots b_{k}}=(L) \overbrace{\left(A_{0}+A_{1}\right)\left(A_{0}^{\prime}+A_{1}^{\prime}\right)\left(A_{0}+A_{1}\right) \cdots}^{k+1} \mid R)=(L \mid R)=1 .
$$

\section{Two-point correlation functions}

Since the matrices $\mathbf{A}, \mathbf{A}^{\prime}$ are finitely dimensional, numerous physically interesting quantities can now be expressed explicitly. The simplest example is the 2-point densitydensity correlation function at different times but at the same position,

$$
C(t)=\langle\rho(0,0) \rho(0, t)\rangle-\langle\rho(0,0)\rangle^{2},
$$

where $\rho(x, t)$ is the density at position $x$ and time $t$, and $\langle\cdot\rangle$ is the expectation value in the (horizontal) stationary state. The correlation function is the $x=0$ part of the spatio-temporal correlation function computed in [14, but here the underlying (horizontal) equilibrium state is richer and not limited to the maximum entropy (infinite temperature) state.

For simplicity, let us consider even times $t=2 \mathrm{~m}$. Then, the correlation function takes the following matrix-product form,

$$
\begin{aligned}
C(2 m) & =\left(L|A_{1} \overbrace{\left(A_{0}^{\prime}+A_{1}^{\prime}\right)\left(A_{0}+A_{1}\right) \cdots\left(A_{0}^{\prime}+A_{1}^{\prime}\right)}^{2 m-1} A_{1}| R\right)-\left(L\left|A_{1}\right| R\right)^{2} \\
& =\left(L\left|A_{1}\left(A_{0}^{\prime}+A_{1}^{\prime}\right) \mathcal{T}^{m-1} A_{1}\right| R\right)-\frac{\left(p_{l}+p_{r}\right)^{2}}{(1+p l+p r)^{2}},
\end{aligned}
$$

where $\mathcal{T}$ is a temporal transfer matrix, i.e. the product of sums of both types of MPA matrices, $\mathcal{T}=\left(A_{0}+A_{1}\right)\left(A_{0}^{\prime}+A_{1}^{\prime}\right)$, as introduced earlier. The correlation function decays 
exponentially, with the exact form given by

$C(2 m)=\frac{\left(\Lambda_{2}^{m}-\Lambda_{3}^{m}\right)\left(\left(p_{l}+p_{r}\right)^{2}-p_{l} p_{r}\left(1+p_{l}+p_{r}\right)\right)+\left(\Lambda_{2}^{m-1}-\Lambda_{3}^{m-1}\right) p_{l} p_{r}\left(p_{l}+p_{r}\right)}{\left(1+p_{l}+p_{r}\right)^{2}\left(\Lambda_{3}-\Lambda_{2}\right)}$,

where $\Lambda_{2,3}$ are the subleading eigenvalues of the matrix $\mathcal{T}$, 9

$$
\Lambda_{2,3}=-\frac{1}{2}\left(p_{l}+p_{r}-p_{l} p_{r} \pm \sqrt{\left(p_{l}+p_{r}-p_{l} p_{r}\right)^{2}-4 p_{l} p_{r}}\right) .
$$

In the maximum entropy state, i.e. $\xi=\omega=1$ or $p_{l}=p_{r}=\frac{1}{2}$, the correlation function can be recast as

$$
C^{\infty}(2 m)=2^{-3 m-4}\left(\left(-1-\frac{\mathrm{i}}{\sqrt{7}}\right)(-3-\mathrm{i} \sqrt{7})^{m}+\left(-1+\frac{\mathrm{i}}{\sqrt{7}}\right)(-3+\mathrm{i} \sqrt{7})^{m}\right),
$$

which agrees with the result of 14$]$.

\section{Probabilities of time-configurations and the absence of decoupling of timescales in RCA54}

Even though the 2-point correlation function is a generalization of the previous result [14, the true advantage of the MPA is the ability to easily express multi-point correlation functions of observables at one specific location in space, as introduced in Eq. (1). Explicitly, for $t_{1} \leq t_{2} \leq t_{3} \ldots \leq t_{k}$, the multi-time correlation function of onesite observables $a_{1}, a_{2}, \ldots a_{k}$ can be expressed in terms of components of the time-state vector $\mathbf{q}$ as

$$
C_{a_{1}, a_{2}, \ldots, a_{k}}\left(t_{1}, t_{2}, \ldots, t_{k}\right)=\sum_{s_{0}, s_{1}, s_{2}, \ldots, s_{t_{k}}} q_{s_{0} s_{1} s_{2} \ldots s_{t_{k}}} a_{1}\left(s_{t_{1}}\right) a_{2}\left(s_{t_{2}}\right) \cdots a_{t_{k}}\left(s_{t_{k}}\right),
$$

where $a_{j}\left(s_{j}\right)$ is the value of the observable $a_{j}$, associated to the one-site configuration $s_{j}$.

The probabilities of time configurations can be understood as extreme cases of multi-time correlation functions, when only one of the terms in the sum (47) has a nonzero prefactor. They can be alternatively recast in an equivalent (non-MPA) form as

$$
q_{s_{1}, s_{2}, \cdots, s_{k}}=\delta_{n_{1}, 0} \frac{\left(1-p_{l}\right)^{n_{2}}\left(1-p_{r}\right)^{n_{3}} p_{r}^{n_{4}} p_{l}^{n_{5}}}{1+p_{l}+p_{r}},
$$

with $\left\{n_{j}, j=1, \ldots, 5\right\}$ being a set of integer coefficients that characterize the number of left and right movers in the configuration $\left(s_{1}, s_{2}, \ldots, s_{k}\right)$,

$$
\begin{aligned}
& n_{1}=\sum_{j=2}^{k-1} s_{j}\left(1-\left(s_{j-1}-s_{j+1}\right)^{2}\right), \quad n_{2}=\sum_{j=1}^{\lfloor k / 2\rfloor}\left(1-s_{2 j-1}\right)\left(1-s_{2 j}\right), \\
& n_{3}=\sum_{j=1}^{\lfloor(k-1) / 2\rfloor}\left(1-s_{2 j}\right)\left(1-s_{2 j+1}\right) . \quad n_{4}=\sum_{j=1}^{\lfloor k / 2\rfloor} s_{2 j-1} s_{2 j}+\bmod (k+1,2)\left(1-s_{k-1}\right) s_{k}, \\
& n_{5}=s_{1}\left(1-s_{2}\right)+\sum_{j=1}^{\lfloor(k-1) / 2\rfloor} s_{2 j} s_{2 j+1}+\bmod (k, 2)\left(1-s_{k-1}\right) s_{k} .
\end{aligned}
$$

I Note that this holds only for $\Lambda_{2} \neq \Lambda_{3}$. If the subleading eigenvalues are degenerate, the exact form of the correlation function is slightly changed, but asymptotically the decay is still exponential. 
The expression (48) is equivalent to the appropriate component of the time vector (37), which can be straightforwardly checked.

In principle, the result above gives access to the full distribution of timescales associated with the dynamics of a single site, when tracing out the rest of the system. This information is particularly important in systems that display complex dynamics and are therefore likely to exhibit non-trivial distributions of local times. As a particular example we consider the distributions of exchange and persistence times in the RCA54. The definitions are those used in the literature on supercooled liquids; see [16, 17]. The exchange time is the time between two events, for example a spin flip.The persistence time is the time to the next event, starting from an arbitrary time, i.e. not conditioned on the previous event having occurred at the start of the time segment under consideration.

If the statistics of events is Poissonian, persistence and exchange times are equally (and exponentially) distributed. In general, however, when there are non-trivial correlations between events, both types of times measure different properties of the underlying process and their distributions differ. For example, in stochastic kinetically constrained models (KCMs) of glasses, like the Fredrickson-Andersen (FA) [18, 19, or the East model [19,20], the two timescales behave very differently, with the average persistence time growing much faster with the decreasing temperature than the average exchange time. This phenomenon is known as transport decoupling [16, 17]. The reason is that the dynamics of these stochastic KCMs is intermittent, and when projected to a single site - in analogy to what is done here for the RCA54 cellular automaton it leads to the bunching of flip events on the site. In this case, the exchange time is dominated by the bunched events, while the persistence time is dominated by the long stretches between bunches. It is interesting to consider the possibility of timescale decoupling in the RCA54, as the dynamical rule (2) imposes a constraint that is similar to that of the FA model (flips can only occur if at least one nearest neighbour is excited). Furthermore, the RCA54 displays dynamical large-deviation transitions [13 similar to those of the FA model [21], which indicates the presence of non-trivial fluctuations in the trajectories of the dynamics.

For the RCA54 we define the exchange time as the time between two consecutive observed particles (excitations) in a time configuration, and the persistence time as the time before the next particle, if we start observing the state at some point. More precisely, we can define the distribution functions of exchange and persistence times and compute them from our explicit form of the time vector in the RCA54. The probability of observing an exchange time equal to $t, p_{\mathrm{E}}(t)$, can be simply expressed in terms of probabilities of time configurations of the form $100 \ldots 01$,

$$
p_{\mathrm{E}}(t) \propto \frac{1}{2}(q_{1}^{1 \underbrace{00 \ldots 0}_{t} 1}+q_{01} \underbrace{00 \ldots 0}_{t} 1+q_{11} \underbrace{00 \ldots 0}_{t} 1), \quad \sum_{t=1}^{\infty} p_{\mathrm{E}}(t)=1,
$$

where we average over the even/odd starting positions of the time configuration. Note that the second term can be dropped, since the subsequence 010 is forbidden. Similarly, the probability of persistence time being equal to $t, p_{\mathrm{P}}(t)$, is, up to normalization, equal 
to

$$
p_{\mathrm{P}}(t) \propto \frac{1}{2}\left(q_{t}^{q_{0}^{00 \ldots 0} 1}+q_{0}^{000 \ldots 0} 1+q_{t}^{100 \ldots 0} 1\right), \quad \sum_{t=1}^{\infty} p_{\mathrm{P}}(t)=1 .
$$

The two distributions are related by

$$
p_{\mathrm{P}}(t)-p_{\mathrm{P}}(t+1)=\frac{p_{\mathrm{E}}(t)}{\left\langle t_{\mathrm{E}}\right\rangle},
$$

which is the discrete-time version of the usual relation between persistence and exchange distributions in continuous-time dynamics. Here, $\left\langle t_{\mathrm{E}}\right\rangle,\left\langle t_{\mathrm{P}}\right\rangle$ are the average exchange and persistence times; $\left\langle t_{\mathrm{E} / \mathrm{P}}\right\rangle=\sum_{t=1}^{\infty} t p_{\mathrm{E} / \mathrm{P}}(t)$.

Using the simplified expressions for probabilities (48), it is easy to see that the exchange and persistence time distributions reduce to the following form:

$$
\begin{aligned}
& p_{\mathrm{E}}(t)=\left\{\begin{array}{lll}
\frac{p_{l}^{2}\left(1-p_{r}\right)+p_{r}^{2}\left(1-p_{l}\right)}{p_{l}+p_{r}}\left(\left(1-p_{l}\right)\left(1-p_{r}\right)\right)^{t / 2-1}, & t \equiv 0 & (\bmod 2), \\
\frac{2 p_{l} p_{r}}{p_{l}+p_{r}}\left(\left(1-p_{l}\right)\left(1-p_{r}\right)\right)^{(t-1) / 2}, & t \equiv 1 & (\bmod 2),
\end{array}\right. \\
& p_{\mathrm{P}}(t)=\left\{\begin{array}{lll}
\frac{p_{l}\left(1-p_{r}\right)+p_{r}\left(1-p_{l}\right)}{2}\left(\left(1-p_{l}\right)\left(1-p_{r}\right)\right)^{t / 2-1}, & t \equiv 0 & (\bmod 2), \\
\frac{p_{l}+p_{r}}{2}\left(\left(1-p_{l}\right)\left(1-p_{r}\right)\right)^{(t-1) / 2}, & t \equiv 1 & (\bmod 2) .
\end{array}\right.
\end{aligned}
$$

If the parameters $p_{l}, p_{r}$ coincide, the two distributions are the same. Otherwise they differ, but the deviations from the Poissonian statistics are small and can be understood as a consequence of short-range correlations of the time state. Specifically, computing the average exchange and persistence times,

$$
\left\langle t_{\mathrm{E}}\right\rangle=\frac{2}{p_{l}+p_{r}}, \quad\left\langle t_{\mathrm{P}}\right\rangle=\frac{4-p_{l}-p_{r}}{2\left(p_{l}+p_{r}-p_{l} p_{r}\right)},
$$

we observe that their ratio is bounded from top and bellow as

$$
1 \leq \frac{\left\langle t_{\mathrm{E}}\right\rangle}{\left\langle t_{\mathrm{P}}\right\rangle} \leq \frac{4}{3}
$$

which indicates that, overall, the two timescales scale similarly with the parameters that control the dynamics, in contrast to what occurs in KCMs like the FA model [16, 17. This also means that, in contrast to stochastic KCMs, to quantify non-trivial correlations in the dynamics of the RCA54 (cf. [13]), it is necessary to consider multi-point correlators both in time and space (not just in time as above).

\section{Conclusion}

We have constructed an explicit matrix-product form of the time state $\mathbf{q}$ in the case of rule 54 reversible cellular automaton. The construction was possible due to the simple structure of the equilibrium state $\mathbf{p}$, which contains left and right movers that are statistically independent, as long as they follow an exclusion rule. The MPA form of the vector $\mathbf{q}$ allows us to efficiently express correlation functions and expectation values of time configurations, i.e. multi-time correlation functions. For instance, we have computed 2-point correlation function and shown that, in the limit $\xi=\omega=1$ 
(i.e. in the maximum entropy state), it agrees with the result of Ref [14]. Furthermore, it is possible to express the probabilities of time configurations in terms of numbers of left and right movers, which enables us to exactly express the distributions of exchange and persistence times and in this way show the absence of timescale decoupling in the model.

The work presented in the paper opens some new research questions. The first one is the generalization of the result to other time-translation invariant (equilibrium) states. The model exhibits an infinite number of conserved quantities, therefore it is possible to construct states $\mathbf{p}$ that are characterized by more than 2 parameters. The question is whether in this case it is still possible to obtain an efficient MPA representation of the time state and what is the dimensionality of the necessary auxiliary space.

Even though the construction of the time state is straightforward, one would like to obtain some algebraic interpretation, for example by finding relations similar to the cubic algebra in 12,13]. They could provide simpler means of extracting physically interesting quantities, as well as contribute additional insight into the integrability structure of the model.

Most prominently, one would like to generalize the result or apply the concept to other lattice dynamical systems. This is intimately connected to the previous question of understanding the solution from an algebraic point of view, which is more robust and does not rely on particularities of the model and the underlying stationary state. Preliminary empirical results suggest that generically, the computational complexity of the time state grows exponentially with time, however one could hope to devise an approximate numerical scheme to efficiently simulate the dynamics (time state) at $x=0$ using the MPA as a variational ansatz 22$]$.

\section{Acknowledgments}

The authors thank Lenart Zadnik for the useful comments on the manuscript. This work has been supported by the European Research Council under the Advanced Grant No. 694544 - OMNES, by the Slovenian Research Agency(ARRS) under the Programme P1-0402, and by the Leverhulme Trust Grant No. RPG-2018-181.

\section{References}

[1] Chandler D and Garrahan J P 2010 Annu. Rev. Phys. Chem. 61 191-217 http://dx.doi.org/ 10.1146/annurev.physchem.040808.090405

[2] Binder K and Kob W 2011 Glassy materials and disordered solids: An introduction to their statistical mechanics (Singapore: World Scientific)

[3] Biroli G and Garrahan J P 2013 J. Chem. Phys. 138 12A301 http://link.aip.org/link/?JCP/ 138/12A301/1

[4] Spohn H 2014 J. Stat. Phys. 154 1191-1227 https://link.springer.com/article/10.1007/ s10955-014-0933-y

[5] Bobenko A, Bordemann M, Gunn C and Pinkall U 1993 Commun. Math. Phys. 158 127-134 https://doi.org/10.1007/BF02097234 
[6] Takesue S 1987 Phys. Rev. Lett. 59(22) 2499-2502 https://link.aps.org/doi/10.1103/ PhysRevLett.59.2499

[7] Gopalakrishnan S 2018 Phys. Rev. B 98(6) 060302 https://link.aps.org/doi/10.1103/ PhysRevB.98.060302

[8] Gopalakrishnan S, Huse D A, Khemani V and Vasseur R 2018 Phys. Rev. B 98(22) 220303 https://link.aps.org/doi/10.1103/PhysRevB.98.220303

[9] Friedman A J, Gopalakrishnan S and Vasseur R 2019 Phys. Rev. Lett. 123(17) 170603 https: //link.aps.org/doi/10.1103/PhysRevLett.123.170603

[10] Prosen T and Mejía-Monasterio C 2016 J. Phys. A: Math. Theor. 49185003 http://stacks. iop.org/1751-8121/49/i=18/a=185003

[11] Inoue A and Takesue S 2018 J. Phys. A: Math. Theor. 51425001 https://doi.org/10.1088\% 2F1751-8121\%2Faadc29

[12] Prosen T and Buča B 2017 J. Phys. A: Math. Theor. 50395002 http://stacks.iop.org/ $1751-8121 / 50 / i=39 / a=395002$

[13] Buča B, Garrahan J P, Prosen T and Vanicat M 2019 Phys. Rev. E 100(2) 020103 https: //link.aps.org/doi/10.1103/PhysRevE.100.020103

[14] Klobas K, Medenjak M, Prosen T and Vanicat M 2019 Commun. Math. Phys. 371 651-688 https://doi.org/10.1007/s00220-019-03494-5

[15] Alba V, Dubail J and Medenjak M 2019 Phys. Rev. Lett. 122(25) 250603 https://link.aps. org/doi/10.1103/PhysRevLett.122.250603

[16] Jung Y, Garrahan J and Chandler D 2004 Phys. Rev. E 69 http://dx.doi.org/061205

[17] Jung Y, Garrahan J P and Chandler D 2005 J. Chem. Phys. 123084509 https://aip. scitation.org/doi/abs/10.1063/1.2001629

[18] Fredrickson G H and Andersen H C 1984 Phys. Rev. Lett. 53 1244-1247 https://link.aps.org/ doi/10.1103/PhysRevLett.53.1244

[19] Garrahan J P 2018 Physica A 504 130-154 https://doi.org/10.1016/j.physa.2017.12.149

[20] Jäckle J and Eisinger S 1991 Z. fur Phys. B 84 115-124 ISSN 0722-3277 http://dx.doi.org/ 10.1007/BF01453764

[21] Garrahan J P, Jack R L, Lecomte V, Pitard E, van Duijvendijk K and van Wijland F 2007 Phys. Rev. Lett. 98(19) 195702 http://dx.doi.org/10.1103/PhysRevLett.98.195702

[22] Schollwöck U 2011 Ann. Phys. 326 96-192 https://doi.org/10.1016/j.aop.2010.09.012

\section{Appendix A. Boundary driving with the steady state that corresponds to the asymptotic probability distribution on a finite chain}

We would like to connect the MPA form of the vectors $\mathbf{p}, \mathbf{p}^{\prime}$, introduced in (23), with an equivalent MPA solution of a boundary driven setup. This amounts to constructing boundary two-site propagators $P^{L / R}$, so that $U_{\mathrm{e} / \mathrm{o}}$, defined as

$U_{\mathrm{e}}=P_{123} P_{345} \cdots P_{2 m-32 m-22 m-1} P_{2 m-12 m}^{R}, \quad U_{\mathrm{o}}=P_{12}^{L} P_{234} P_{456} \cdots P_{2 m-22 m-12 m}$,

map $\mathbf{p}$ to $\mathbf{p}^{\prime}$, and vice versa.

Taking into account the bulk relations (11) and (13), it suffices that the following boundary equations are satisfied,

$\langle l| \mathbf{W}_{1} S=\alpha\left\langle l^{\prime}\right| \mathbf{W}_{1}^{\prime}, \quad P_{12}^{L}\left(\left\langle l^{\prime}\right| \mathbf{W}_{1}^{\prime} \mathbf{W}_{2} S\right)=\gamma\langle l| \mathbf{W}_{1} \mathbf{W}_{2}^{\prime}$,

$\mathbf{W}_{2 m}^{\prime} S\left|r^{\prime}\right\rangle=\delta \mathbf{W}_{2 m}^{\prime}|r\rangle, \quad P_{2 m-12 m}^{R}\left(\mathbf{W}_{2 m-1}^{\prime} S \mathbf{W}_{2 m}^{\prime}|r\rangle\right)=\beta \mathbf{W}_{2 m-1}^{\prime} \mathbf{W}_{2 m}\left|r^{\prime}\right\rangle$

where the parameters $\alpha, \beta, \gamma$ and $\delta$ have to fulfill the condition

$$
\alpha \beta=\frac{\langle l \mid r\rangle}{\left\langle l^{\prime} \mid r^{\prime}\right\rangle}=\frac{1}{\gamma \delta}
$$


Solving these equations, we obtain the following boundary propagators,

$$
P^{R}=\left[\begin{array}{cccc}
1-p_{l} & 1-p_{l} & 0 & 0 \\
p_{l} & p_{l} & 0 & 0 \\
0 & 0 & 0 & 1 \\
0 & 0 & 1 & 0
\end{array}\right], \quad P^{L}=\left[\begin{array}{cccc}
1-p_{r} & 0 & 1-p_{r} & 0 \\
0 & 0 & 0 & 1 \\
p_{r} & 0 & p_{r} & 0 \\
0 & 1 & 0 & 0
\end{array}\right]
$$

with parameters $p_{r}, p_{l}$ corresponding to the conditional probabilities of observing right/left movers as expressed in (27), (26), and the following values of $\alpha, \beta, \gamma, \delta$,

$$
\begin{aligned}
\alpha & =\frac{\lambda+\xi-\xi \omega}{\lambda+\omega-\xi \omega}, & \beta & =\frac{\omega}{\xi}, \\
\gamma & =1, & \delta & =\frac{\xi(\lambda+\omega-\xi \omega)}{\omega(\lambda+\xi-\xi \omega)} .
\end{aligned}
$$

Note that the choice of $P^{R, L}$ is not unique.

The boundary relations A.2 and the solution are simpler than the ones considered in [12], since the boundary vectors do not depend on the configuration of the boundary sites. These states therefore represent a specific subset of solutions found in the previous works $10-12$.

\section{Appendix B. Conditional probabilities of observing subconfigurations}

To prove the relations (24) and 25), we observe that the vectors $\langle l| W_{s_{1}} W_{s_{2}}^{\prime} W_{s_{3}} W_{s_{4}}^{\prime}$ and $\langle l| W_{s_{3}} W_{s_{4}}^{\prime}$ (similarly $W_{s_{1}} W_{s_{2}}^{\prime} W_{s_{3}} W_{s_{4}}^{\prime}|r\rangle$ and $\left.W_{s_{1}} W_{s_{2}}^{\prime}|r\rangle\right)$ are linearly dependent. Explicitly, for every 4-site configuration $\left(s_{1}, s_{2}, s_{3}, s_{4}\right)$ there exist scalar factors $c_{s_{1} s_{2} s_{3} s_{4}}^{(l)}$ and $c_{s_{1} s_{2} s_{3} s_{4}}^{(r)}$, so that the following holds,

$$
\begin{aligned}
& \langle l| W_{s_{1}} W_{s_{2}}^{\prime} W_{s_{3}} W_{s_{4}}^{\prime}=c_{s_{1} s_{2} s_{3} s_{4}}^{(l)}\langle l| W_{s_{3}} W_{s_{4}}^{\prime}, \\
& W_{s_{1}} W_{s_{2}}^{\prime} W_{s_{3}} W_{s_{4}}^{\prime}|r\rangle=c_{s_{1} s_{2} s_{3} s_{4}}^{(r)} W_{s_{1}} W_{s_{2}}^{\prime}|r\rangle,
\end{aligned}
$$

which can be straightforwardly verified by checking all 16 configurations. From here relations (24) and (25) follow directly, since for every even configuration length $2 k$ the left-hand side of the first part of equation (24) can be rewritten as

$$
\begin{aligned}
& \frac{\left\langle l\left|W_{s_{1}} W_{s_{2}}^{\prime} \cdots W_{s_{2 k-3}} W_{s_{2 k-2}}^{\prime} W_{s_{2 k-1}}^{\prime} W_{s_{2 k}}^{\prime}\right| r\right\rangle}{\left\langle l\left|W_{s_{1}} W_{s_{2}}^{\prime} \cdots W_{s_{2 k-3}} W_{s_{2 k-2}}^{\prime}\right| r\right\rangle} \\
& =\frac{c_{s_{1} s_{2} s_{3} s_{4}}^{(l)} \cdots c_{s_{2 k-5} s_{2 k-4} s_{2 k-3} s_{2 k-2}}^{(l)}\left\langle l\left|W_{s_{2 k-3}} W_{s_{2 k-2}}^{\prime} W_{s_{2 k-1}} W_{s_{2 k}}^{\prime}\right| r\right\rangle}{c_{s_{1} s_{2} s_{3} s_{4}}^{(l)} \cdots c_{s_{2 k-5} s_{2 k-4} s_{2 k-3} s_{2 k-2}}^{(l)}\left\langle l\left|W_{s_{2 k-3}} W_{s_{2 k-2}}^{\prime}\right| r\right\rangle} \\
& =\frac{\left\langle l\left|W_{s_{2 k-3}} W_{s_{2 k-2}}^{\prime} W_{s_{2 k-1}} W_{s_{2 k}}^{\prime}\right| r\right\rangle}{\left\langle l\left|W_{s_{2 k-3}} W_{s_{2 k-2}}^{\prime}\right| r\right\rangle},
\end{aligned}
$$

where the first equality follows from the repeated application of the relation (B.1). From Eq. (B.2) we analogously obtain

$$
\frac{\left\langle l\left|W_{s_{1}} W_{s_{2}}^{\prime} W_{s_{3}} W_{s_{4}}^{\prime} \cdots W_{s_{2 k-1}} W_{s_{2 k}}^{\prime}\right| r\right\rangle}{\left\langle l\left|W_{s_{3}} W_{s_{4}}^{\prime} \cdots W_{s_{2 k-1}} W_{s_{2 k}}^{\prime}\right| r\right\rangle}=\frac{\left\langle l\left|W_{s_{1}} W_{s_{2}}^{\prime} W_{s_{3}} W_{s_{4}}^{\prime}\right| r\right\rangle}{\left\langle l\left|W_{s_{3}} W_{s_{4}}^{\prime}\right| r\right\rangle} .
$$

Note that the pair of relations dealing with probabilities $p_{s}^{\prime}$ follows trivially after the exchange $\xi \leftrightarrow \omega$. 